\title{
Erratum
}

\section{A short proof of finiteness of Murty's principal pivoting algorithm}

\section{Jiri Rohn}

Faculty of Mathematics and Physics, Charles University, Malostranske nam. 25, 11800 Prague, Czechoslovakia

[Mathematical Programming 46 (1990) 255-256]

In the formulation of the linear complementarity problem

$$
\begin{aligned}
& y=M z+q, \\
& y^{\top} z=0, \\
& y \geqslant 0, \quad z \geqslant 0,
\end{aligned}
$$

equation (2) should be replaced by

$$
y_{i} z_{i}=0 \quad(i=1, \ldots, n)
$$

since the proof of the lemma on p. 255 actually uses $\left(2^{\prime}\right)$, not (2) (condition (3) is not assumed to hold there, so that $(2)$ and $\left(2^{\prime}\right)$ are not equivalent in the context). After this change, all the subsequent results are correct. 